\title{
Desempeño de la presepsina como biomarcador temprano de sepsis en un hospital de alta complejidad en Medellín, Colombia
}

\author{
Performance of presepsin as early biomarker of sepsis \\ in a high complexity hospital from Medellin, Colombia
}

Tatiana J. Castaño-Sepúlveda MB', Mary L. Arévalo-Ruano MB², J. Andres CastilloRamírez MB $^{3}$, M. Gabriela Becerra-Argote $M{ }^{4}{ }^{4}$, Sigifredo Ospina-Ospina MD $^{5}$

\begin{abstract}
Introducción: la presepsina es el subtipo soluble de la glicoproteína CD14 expresada en la superficie de membrana de los monocitos y los macrófagos; molécula importante en el proceso inflamatorio. Varios estudios sugieren realizar su medición para la identificación temprana de la sepsis. Objetivo: determinar el desempeño diagnóstico y pronóstico de la presepsina en pacientes con sepsis clínica de un hospital de alta complejidad en Medellín, Colombia. Materiales y métodos: se realizó un estudio descriptivo prospectivo de cohorte única en 60 pacientes con diagnóstico de sepsis clínica durante marzo y diciembre de 2012. La presepsina se midió al momento del diagnóstico, a las 24 y 72 horas; la proteína $C$ reactiva, la procalcitonina y el hemocultivo sólo al momento del diagnóstico. Se utilizaron herramientas de estadística descriptiva para la caracterización de la población y análisis bivariado para la comparación entre medianas. Resultados: El 98,3\% de los pacientes tuvieron valores de presepsina sugestivos de sepsis, observándose un valor significativamente más alto en los pacientes sin mejoría (mayor que $700 \mathrm{pg} / \mathrm{mL}$ ). Se observaron valores de presepsina mayores que $1.000 \mathrm{pg} / \mathrm{mL}$ a las 0 y 72 horas del diagnóstico en los pacientes que murieron, pero no se observaron diferencias significativas en comparación con los que no murieron. La proteína $C$ reactiva y la procalcitonina mostraron valores aumentados en la mayoría de los pacientes. Conclusiones: los hallazgos de este estudio se relacionan con lo encontrado en otros estudios donde concluyen que la presepsina es un buen marcador de sepsis, con un importante valor pronóstico y mayor especificidad que otros biomarcadores tradicionales.
\end{abstract}

Palabras clave: sepsis, biomarcadores, diagnóstico, antígenos CD14.

Castaño-Sepúlveda TJ, Arévalo-Ruano ML, Castillo-Ramírez JA, Becerra-Argote MG, Ospina-Ospina S. Desempeño de la presepsina como biomarcador temprano de sepsis en un hospital de alta complejidad en Medellín, Colombia. Medicina \& Laboratorio 2017; 23: 85-94.

${ }^{1}$ Microbióloga y Bioanalista. Bacterióloga sección Biología Molecular e Inmunología de Trasplantes,

Hospital Universitario de San Vicente Fundación. Medellín, Colombia. Correspondencia: Calle 64 N. ${ }^{\circ}$ 51D - 155. Teléfono: 5744441333 ext. 3106 - 2558. Correo electrónico: tjcs@sanvicentefundacion.com

${ }^{2}$ Microbióloga y Bioanalista. Bacterióloga IDEME S.A. Pasto, Colombia.

${ }^{3}$ Microbiólogo y Bioanalista. Auxiliar de investigación, Escuela de Microbiología, Universidad de Antioquia. Medellín, Colombia.

${ }^{4}$ Bacterióloga y Laboratorista Clínica, MSc en Epidemiología. Bacterióloga, epidemióloga Laboratorio Clínico y Unidad de Investigaciones, Hospital Universitario de San Vicente Fundación. Medellín, Colombia.

${ }^{5}$ Médico, Microbiólogo y Parasitólogo Médico, y Epidemiólogo. Jefe del Laboratorio Clínico, Epidemiología Hospitalaria y Unidad de Investigaciones, Hospital Universitario de San Vicente Fundación. Medellín, Colombia.

Conflicto de intereses: los autores declaran que no tienen conflicto de intereses Medicina \& Laboratorio 2017; 23: 85-94

Módulo 19 (Investigación), número 53. Editora Médica Colombiana S.A. 2017@

Recibido el 20 de febrero de 2017; aceptado el 28 de febrero de 2017 
a sepsis es una inflamación sistémica corporal potencialmente fatal, la cual normalmente es causada por una infección grave [1]. Esta entidad tiene una alta prevalencia a nivel mundial y es uno de los diagnósticos de ingreso más frecuente de los pacientes en los servicios de urgencias y cuidados intensivos [2]. En los últimos 30 años la incidencia mundial de la sepsis ha aumentado un $13,7 \%$ anual y se han estimado alrededor de 18.000.000 de casos, de los cuales 5.000.000 mueren [3-6]. Para Colombia, en el año 2007, un estudio multicéntrico en diez hospitales universitarios reveló una incidencia de 2.681 casos de pacientes con criterio de sepsis, con una mortalidad del 19\% [7].

El diagnóstico de la sepsis está basado principalmente en los hallazgos clínicos y en parámetros de laboratorio como el hemocultivo, el cual es considerado el estándar de referencia para la confirmación de la sepsis en un paciente con signos y síntomas sugestivos [8]; sin embargo, una limitante de esta prueba es su sensibilidad, la cual puede variar desde un $30 \%$ hasta un $80 \%$ dependiendo de diferentes factores biológicos e inherentes al procedimiento [9].

Además, los hemocultivos requieren de cierto tiempo para obtener un resultado, lo cual puede resultar crítico para instaurar rápidamente una terapia adecuada; sumado a que se presenta un porcentaje importante de contaminación [10]. El tratamiento de la sepsis es frecuentemente fundamentado en la experiencia clínica y criterio del médico tratante, lo cual contribuye al incremento del riesgo de resistencia a los antibióticos y de los costos en el sistema de salud [11].

Con base en lo anterior, es evidente la necesidad de encontrar métodos diagnósticos alternativos que sean más eficientes y oportunos, como es el caso de los marca- dores séricos. Entre los más utilizados en el diagnóstico y seguimiento de la sepsis se encuentran la proteína $C$ reactiva y la procalcitonina. La primera es un biomarcador utilizado para la detección de procesos inflamatorios, que participa en diversos procesos inmunomoduladores y se eleva rápidamente en respuesta a estímulos infecciosos y disminuye con el cese de los mismos. Por su parte, la procalcitonina aumenta en respuesta a la estimulación bacteriana y luego, cuando dicho estímulo desaparece, comienza a decaer [12].

Estos marcadores también se pueden aumentar durante procesos no infecciosos como traumatismos, intervenciones quirúrgicas complejas y tratamientos farmacológicos que estimulan la liberación de citoquinas proinflamatorias, lo que genera dudas sobre su papel en el diagnóstico específico de la sepsis [13]. Lo anterior genera una gran incertidumbre en los médicos tratantes, por lo que se buscan nuevas pruebas que contribuyan a mejorar el proceso diagnóstico.

La molécula SCD14-ST, también conocida como presepsina, es el subtipo soluble de la glicoproteína CD14, la cual se expresa en la superficie de la membrana de los monocitos y los macrófagos y desempeña un rol importante en la iniciación de la cascada proinflamatoria [14]. Estudios previos la describen como un marcador de sepsis promisorio, altamente específico, debido a su mecanismo de producción en el proceso de la fagocitosis bacteriana; además, se plantea que puede tener un desempeño diagnóstico y pronóstico mayor que la proteína $\mathrm{C}$ reactiva, la procalcitonina y la interleuquina-6 (IL-6), que puede reflejar incluso el grado de severidad $[15,16]$, con un alto valor predictivo de la mortalidad y que se puede emplear también para el seguimiento de la enfermedad y de la respuesta terapéutica [17]. 
En vista de la limitada información disponible en el medio acerca de este reciente biomarcador de sepsis, el propósito de este estudio fue determinar la utilidad diagnóstica y pronostica de la presepsina como marcador de sepsis en pacientes hospitalizados en el Hospital Universitario de San Vicente Fundación de Medellín, Colombia, para evaluar su posible implementación como prueba de rutina en el laboratorio clínico.

\section{Materiales y métodos}

\section{Tipo y población de estudio}

Se realizó un estudio descriptivo prospectivo de cohorte única con 60 pacientes atendidos entre marzo y diciembre del año 2012 en el Hospital Universitario de San Vicente Fundación de la ciudad de Medellín, Colombia, con diagnóstico clínico de sepsis clínica y a quienes durante el proceso diagnóstico se les realizó hemocultivo, proteína $C$ reactiva y procalcitonina. Aquellos pacientes que tuvieron los exámenes mencionados previos al diagnóstico no fueron incluidos.

Los criterios de exclusión fueron: la no aceptación del paciente a participar en el estudio y no disponer de la información completa de la historia clínica del paciente.

El muestreo fue realizado por conveniencia por parte de un médico microbiólogo del laboratorio clínico del hospital.

\section{Medición de biomarcadores serológicos}

La determinación cuantitativa de la procalcitonina se realizó empleando la técnica de inmunoensayo fluorescente ligado a enzima (ELFA) mediante el analizador Vidas BR-A-H-M-S ${ }^{T M}$ (BioMérieux, Marcy-l'Étoile, Francia) $[18,19]$. Por su parte, la evaluación cuantitativa de la proteína $C$ reactiva se realizó por el método de inmunoturbidimetria cuantitativa en el sistema ARCHITECT (Abbott Laboratories, Chicago, Estados Unidos) $[20,21]$. La medición de la presepsina se realizó por el ensayo inmunoenzimático quimioluminiscente en el analizador Pathfast ${ }^{\circ}$ (Mitsubishi Chemical Medience Corporation, Japón) [15,22].

La medición de la proteína $C$ reactiva y la procalcitonina se realizó una sola vez al momento de la atención médica, mientras que a todos los pacientes se les efectuó medición de la presepsina en tres instantes: al momento del diagnóstico, a las 24 y a las 72 horas.

Los valores de referencia para cada uno de los biomarcadores serológicos se definieron con base en las instrucciones del fabricante y la revisión de la literatura [15,18-22] (véase tabla 1).

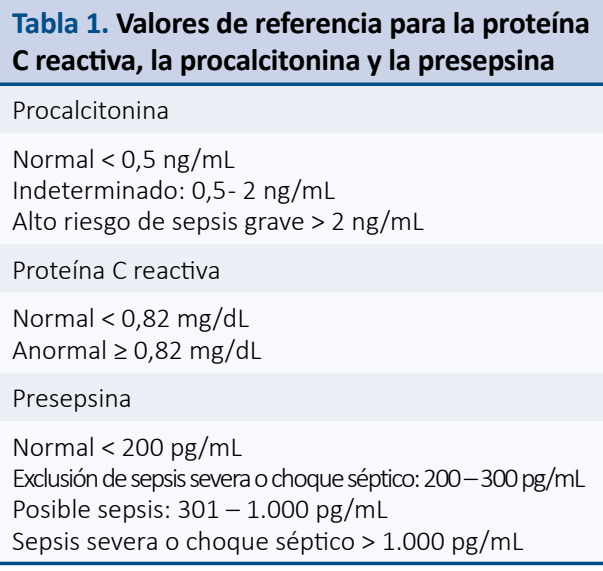

\section{Información}

\section{demográfica y clínica}

La información relacionada con las variables objeto del estudio fue tomada a partir de la historia clínica de cada paciente y de los resultados del laboratorio clínico. El diagnóstico de sepsis clínica fue determinado según la guía de práctica clínica institucional, la cual está basada en los criterios del Cole- 
gio Americano de Médicos del Tórax/Sociedad de Cuidados Intensivos Críticos (ACCP) SCCM; del inglés, American College of Chest Physicians/Society of Critical Care Medicine) de Estados Unidos [2].

Los datos obtenidos fueron organizados en un formulario y las variables consignadas fueron: edad, sexo, origen de la sepsis, resultado del hemocultivo, resultados de los marcadores de sepsis y estado del paciente a los 14 días del diagnóstico.

\section{Análisis de la información}

La tabulación de la información se realizó en Excel $2010^{\circ}$ (Microsoft Corporation, Washington, Estados Unidos). Posteriormente, los datos se analizaron en el programa IBM SPSS para Windows, versión 16.0 (IBM Corporation, Nueva York, Estados Unidos). La descripción de la población de estudio y sus características se hizo mediante estadística descriptiva, entre estas, la distribución de frecuencias absoluta y relativa para cada una de las categorías de las variables. Para los datos cuantitativos se calculó el valor mediano con su respectivo rango intercuartílico. Para los análisis bivariados se utilizó la prueba de U de Mann-Whitney y un valor de significancia estadística menor que $0,05(p<0,05)$. Se realizaron comparaciones entre los resultados de las pruebas y los resultados del hemocultivo, así como con el estado del paciente a los 14 días del diagnóstico.

\section{Consideraciones éticas}

Para la realización de este proyecto se tomó consentimiento informado verbal a cada uno de los pacientes, se contó con el permiso institucional para su ejecución y se garantizó en todo momento la confidencialidad de la información.
Este estudio fue aprobado por el comité de ética del Hospital Universitario de San Vicente Fundación según la resolución 008430 de 1993 del Ministerio de Salud de Colombia, como una investigación sin riesgo pues utilizó información proveniente de fuente secundaria y no se realizaron intervenciones en el paciente.

\section{Resultados}

Se estudiaron 60 pacientes con diagnóstico de sepsis clínica, cuya edad promedio fue de 40,9 años (desviación estándar $=25,7$ ). La edad mínima fue de 0 años y la máxima de 94 años, con un leve predominio del sexo masculino (véase tabla 2 ).

\begin{tabular}{|c|c|c|}
\hline Características generales & N..$^{\circ}$ & $\%$ \\
\hline \multicolumn{3}{|l|}{ Edad } \\
\hline $0-15$ & 11 & 18,3 \\
\hline $16-30$ & 15 & 25,0 \\
\hline $31-45$ & 11 & 18,3 \\
\hline $46-60$ & 6 & 10,0 \\
\hline $61-75$ & 12 & 20,0 \\
\hline $76-90$ & 3 & 5,0 \\
\hline 91 o más & 2 & 3,3 \\
\hline \multicolumn{3}{|l|}{ Sexo } \\
\hline Masculino & 32 & 53,3 \\
\hline Femenino & 28 & 46,7 \\
\hline \multicolumn{3}{|l|}{ Hospitalización } \\
\hline Unidad de cuidados intensivos & 10 & 16,7 \\
\hline Otras & 50 & 83,3 \\
\hline
\end{tabular}

El origen predominante de la sepsis fue la infección a nivel urinario con el 23,3\% (14/60), mientras que los menos frecuentes fueron las infecciones del sistema nervioso central, origen biliar y osteoarticulares, cada uno con un $1,7 \%(1 / 60)$ de frecuencia (véase figura 1 ). 


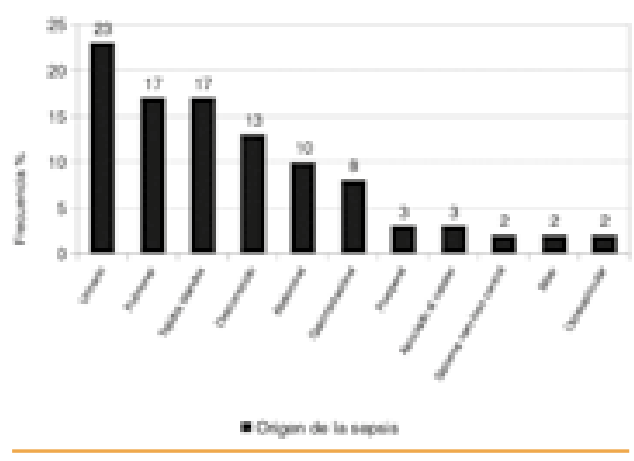

Figura 1. Frecuencia del origen de sepsis en la población de estudio $(n=60)$.

Al momento del diagnóstico de sepsis los biomarcadores proteína $\mathrm{C}$ reactiva y procalcitonina se encontraron con valores aumentados en el $95,0 \%(57 / 60)$ y el $65,0 \%(39 / 60)$ de los pacientes, respectivamente, mientras que la presepsina se encontró elevada en el $98,3 \%$ $(59 / 60)$ de ellos. La mediana general de los diferentes marcadores al momento del diagnóstico fue: proteína $C$ reactiva $12,86 \mathrm{mg} / \mathrm{dL}$ (rango intercuartílico $=4,9-22,9)$, procalcitonina $1,42 \mathrm{ng} / \mathrm{mL}$ (rango intercuartílico = $0,28-6,65$ ) y presepsina $1.016 \mathrm{pg} / \mathrm{mL}$ (rango intercuartílico $=617,2-3.201,2$ ); este último con otras dos mediciones posteriores ( 24 y 72 horas) (véase tabla $\mathbf{3}$ ).

\begin{tabular}{|c|c|c|}
\hline $\begin{array}{l}\text { Niveles de presepsina } \\
\text { (pg/mL) }\end{array}$ & Mediana & $\begin{array}{l}\text { Rango } \\
\text { intercuartílico }\end{array}$ \\
\hline 0 horas & $1.016,5$ & $617,2-3.201,2$ \\
\hline 24 horas & 843 & $436,0-3.617,0$ \\
\hline 72 horas & 532 & $303,5-3.092,0$ \\
\hline
\end{tabular}

El 20\% (12/60) de los pacientes estudiados tuvieron hemocultivos positivos, cuyos microorganismos más frecuentes fueron Escherichia coli (4/20) y Staphylococcus aureus (4/20).
La mediana de los tres marcadores séricos, al momento del diagnóstico clínico de sepsis, según el resultado del hemocultivo, se presenta en la tabla 4; los niveles de presepsina en las tres mediciones en el tiempo $(0$, 24 y 72 horas) según el resultado del hemocultivo en la tabla 5.

No se encontraron diferencias significativas entre los resultados de la presepsina de los pacientes que murieron y los que no murieron; sin embargo, se observó en los pacientes que fallecieron un aumento importante en la mediana de los valores de presepsina a las 72 horas respecto a los valores basales y a las 24 horas del diagnóstico. Además, se presentaron diferencias significativas $(p<0,05)$ en los valores medianos de la presepsina a las 72 horas del diagnóstico clínico entre los pacientes que tuvieron mejoría clínica y los que no (véase tabla 6 ).

El 98,3\% (59/60) de los pacientes tuvo valores de presepsina sugestivos de sepsis de acuerdo a los valores de referencia del estuche utilizado. El $100 \%$ de los pacientes que murieron o no mejoraron tuvo valores de presepsina por encima de $300 \mathrm{pg} / \mathrm{mL}$.

\section{Discusión}

La identificación temprana de la sepsis y la implementación de una terapia adecuada mejora los resultados clínicos de los pacientes y reduce la mortalidad asociada. De esta manera, disminuir el tiempo del diagnóstico de la sepsis es un aspecto crítico para el manejo de los pacientes que la padecen [23]. En el presente estudio se documentó el desempeño de un biomarcador emergente, la presepsina, como posible ayuda diagnóstica de la sepsis en el contexto de un hospital de alta complejidad. 
Tabla 4. Niveles de los biomarcadores en los pacientes según resultado de los hemocultivos ( $n=60)$

\begin{tabular}{llll}
\hline \multicolumn{1}{c}{ Biomarcadores séricos } & \multicolumn{2}{c}{$\begin{array}{c}\text { Hemocultivo } \\
\text { [mediana (rango intercuartílico)] }\end{array}$} & Valor $\boldsymbol{p}^{*}$ \\
& \multicolumn{1}{c}{ Positivo $(\mathbf{n = 1 2})$} & Negativo $(\mathbf{n = 4 8 )}$ & \\
\hline Proteína C reactiva $(\mathrm{mg} / \mathrm{dL})$ & $20,7(14,71-30,06)$ & $8,8(4,22-22,15)$ & 0,006 \\
Procalcitonina $(\mathrm{ng} / \mathrm{mL})$ & $6,1(0,685-32,42)$ & $1,2(0,255-5,64)$ & 0,084 \\
Presepsina $(\mathrm{pg} / \mathrm{mL})$ & $1.206,5(756,5-2.073,5)$ & $100,0(629,5-5.306,0)$ & 0,796 \\
*U-Man Withney & & & \\
\hline
\end{tabular}

\begin{tabular}{|c|c|c|c|}
\hline \multirow{2}{*}{$\begin{array}{c}\text { Presepsina }(\mathrm{pg} / \mathrm{mL}) \text { en cada tiempo } \\
\text { de medición }\end{array}$} & \multicolumn{2}{|c|}{$\begin{array}{c}\text { Hemocultivo } \\
\text { [mediana (rango intercuartílico) }\end{array}$} & \multirow{2}{*}{$\begin{array}{c}\text { Valor } \\
p^{*}\end{array}$} \\
\hline & Positivo ( $n=12$ ) & Negativo $(n=48)$ & \\
\hline 0 horas & $1.206,5(690-2.936,0)$ & $100,1(583,0-3.385,0)$ & 0,796 \\
\hline 24 horas & $1.119,0(545,5-4.325,0)$ & $780,0(421,2-2.812,5)$ & 0,332 \\
\hline 72 horas & $1.191,5(442,7-5.055,0)$ & $488,5(271,2-2.998,5)$ & 0,057 \\
\hline
\end{tabular}

Tabla 6. Resultados de la presepsina según desenlace y evolución en la población de estudio ( $\mathrm{n}=60)$

\begin{tabular}{|c|c|c|c|c|c|c|}
\hline \multirow{2}{*}{$\begin{array}{l}\text { Presepsina }(\mathrm{pg} / \mathrm{mL}) \\
\text { en cada tiempo de } \\
\text { medición }\end{array}$} & \multicolumn{2}{|c|}{$\begin{array}{l}\text { Pacientes } \\
\text { [mediana (rango intercuartílico)] }\end{array}$} & \multirow[t]{2}{*}{ Valor $p^{*}$} & \multicolumn{2}{|c|}{$\begin{array}{l}\text { Pacientes } \\
\text { [mediana (rango intercuartílico)] }\end{array}$} & \multirow[t]{2}{*}{ Valor $p^{*}$} \\
\hline & $\begin{array}{l}\text { Fallecidos } \\
(n=9)\end{array}$ & $\begin{array}{l}\text { No fallecidos } \\
(n=51)\end{array}$ & & $\begin{array}{l}\text { Con mejoría } \\
(n=42)\end{array}$ & $\begin{array}{l}\text { Sin mejoría } \\
(n=15)\end{array}$ & \\
\hline 0 horas & & $\begin{array}{l}1.026(590,0- \\
3.112,0)\end{array}$ & 0,55 & $\begin{array}{l}945(562,5- \\
2.740,7)\end{array}$ & $\begin{array}{l}1.378(929,2 \\
-4.643,0)\end{array}$ & 0,190 \\
\hline 24 horas & $\begin{array}{l}762 \\
(495,5- \\
10.128,0)\end{array}$ & $\begin{array}{l}898(431,0- \\
2.987,0)\end{array}$ & 0,425 & $\begin{array}{l}721(406,5- \\
2.497,5)\end{array}$ & $\begin{array}{l}2.026(786,0 \\
-4.182,0)\end{array}$ & 0,101 \\
\hline 72 horas & $\begin{array}{l}3.336 \\
(345,5- \\
9.621,5)\end{array}$ & $\begin{array}{l}478(296,0- \\
2.427,0)\end{array}$ & 0,15 & $\begin{array}{l}435(277,5- \\
1.957,7)\end{array}$ & $\begin{array}{l}726(542,0- \\
5.621,0)\end{array}$ & 0,027 \\
\hline *U-Man Withney & & & & & & \\
\hline
\end{tabular}

La sepsis se origina a partir de focos primarios en diferentes órganos o sistemas como los pulmones, el abdomen, el tracto urinario y los tejidos blandos, o por infección relacionada con el catéter intravascular [2,7,23]. En este estudio el sitio de origen más frecuente de sepsis fue el tracto urinario, seguido de los pulmones y los tejidos blandos, lo que se relaciona con los hallazgos de un estudio realizado en 2007 en un hospital en Colom- bia [24], donde el proceso infeccioso más frecuente fue el del tracto urinario, seguido de la neumonía adquirida en la comunidad. Sin embargo, en el $13,3 \%(8 / 60)$ de los pacientes incluidos en el presente estudio el origen de la sepsis fue desconocido, sin lograrse establecer un foco primario.

Tradicionalmente, el diagnóstico de la sepsis se realiza con base en los criterios clínicos y 
parámetros del laboratorio como la proteína $C$ reactiva, la procalcitonina y el hemocultivo $[8,12]$; sin embargo, estas pruebas no son específicas $y$, por lo tanto, no se pueden interpretar de manera aislada, lo que evidencia la necesidad de estudiar otros posibles marcadores como es el caso de la presepsina.

En este estudio se encontró que la proteína $C$ reactiva aumentaba en la mayoría de los pacientes al momento del diagnóstico clínico, mientras que la procalcitonina aumentaba en una menor proporción de casos, de forma similar a lo obtenido por Julián y colaboradores (2009) [25], quienes además encontraron valores significativamente superiores de ambos marcadores en los pacientes con sepsis respecto a los pacientes con síndrome de respuesta inflamatoria sistémica. En cuanto a la presepsina, en el presente estudio se encontró aumentada en la mayoría de los pacientes, tal como se ha observado en otras investigaciones en las que se compararon los niveles de procalcitonina, proteína $C$ reactiva y presepsina [26-30].

La mediana de los valores de proteína $C$ reactiva en los pacientes del presente estudio fue de $12,8 \mathrm{mg} / \mathrm{dL}$, la cual es similar a las concentraciones registradas por Povoa y colaboradores (2005) [31] en pacientes con sepsis (15,2 \pm $8,2 \mathrm{mg} / \mathrm{dL}$ ). En relación con la mediana de los valores de procalcitonina existe cierta variabilidad entre las diferentes fuentes bibliográficas $[18,19,26,28]$. La mediana de la procalcitonina obtenida en este estudio fue de 1,31 ng/ $\mathrm{mL}$, indicativa, según los valores de referencia de la prueba, de posibilidad de una infección localizada o probable sepsis $[25,26]$.

En cuanto a los valores medianos de la presepsina en los pacientes con diagnóstico de sepsis se ha encontrado una amplia variabilidad en la literatura disponible. Yaegashi y colaboradores (2005) [14], en un estudio con 66 pacientes con sepsis, obtuvieron una media de $220,7 \mathrm{pg} / \mathrm{mL}$, con una diferencia estadísticamente significativa en comparación con los pacientes control. Por su parte, Carpio y colaboradores (2015) [17], en un estudio de 259 pacientes, encontraron una media de presepsina de $2.563 \mathrm{pg} / \mathrm{mL}$. Esta variabilidad en los resultados de la presepsina puede estar relacionada con el tipo de población, el tamaño de la muestra, los diferentes estadios de la sepsis (de bajo grado o severa) e incluso la metodología de medición empleada.

En términos generales, el valor mediano de presepsina en el presente estudio fue de $1.016 \mathrm{pg} / \mathrm{mL}$ (rango intercuartílico: 617,2$3.201,2 \mathrm{pg} / \mathrm{mL}$ ), el cual corresponde a una alta probabilidad de sepsis severa o choque séptico de acuerdo a los valores de referencia definidos por el fabricante de la prueba [22]. También se observó que la mayoría de los pacientes presentaban valores aumentados de presepsina al momento del diagnóstico de sepsis, los cuales disminuyeron a las 12 y 72 horas, relacionándose con el porcentaje de mejoría o curación de la población estudiada, el cual fue del $70 \%$.

En cuanto a la evolución de los pacientes luego de los 14 días del diagnóstico se observó un valor significativamente más alto de presepsina en los pacientes que no mejoraron. Por otro lado, los pacientes que murieron o no mejoraron tuvieron resultados de presepsina mayores que $1.000 \mathrm{pg} /$ $\mathrm{mL}$ al momento del diagnóstico, al igual que en la medición a las 72 horas. Estos hallazgos se relacionan con lo encontrado en otros reportes en los que se concluye que la presepsina es un buen marcador de sepsis severa, con un importante valor pronóstico y con mayor especificidad que otros marcadores [15-17,27]. 
En el estudio realizado por Carpio y colaboradores (2015) [17] se afirma que la presepsina posee un valor pronóstico más confiable que la procalcitonina y la proteína $C$ reactiva y que podría predecir el riesgo de mortalidad precozmente. Asimismo, en dicho trabajo se encontró que la media de los valores de presepsina a las 72 horas del diagnóstico fue mayor en los pacientes que murieron que en los no fallecidos.

Todos los pacientes con hemocultivos positivos en el presente estudio tuvieron valores aumentados de proteína $\mathrm{C}$ reactiva y presepsina, y solo el $75 \%$ de procalcitonina, similar a lo encontrado por Shozushima y colaboradores (2011) [15], donde el rendimiento diagnóstico de la procalcitonina fue bajo, y en concordancia con un estudio realizado en Colombia recientemente, en el que la presepsina fue superior a los marcadores convencionales y al hemocultivo [28]. No se observaron diferencias significativas entre la media de los valores de presepsina en los pacientes con hemocultivos positivos y negativos, lo cual se puede relacionar con el bajo rendimiento diagnóstico de los hemocultivos en pacientes sospechosos y diagnosticados con sepsis debido a otras variables como el uso previo de antibióticos [29]. De acuerdo a los hallazgos de un metanálisis sobre múltiples biomarcadores de sepsis, la sensibilidad en el diagnóstico de esta entidad se obtiene al realizar simultáneamente la medición de presepsina, proteína $\mathrm{C}$ reactiva y procalcitonina [30].

Como limitaciones del presente estudio es importante resaltar que no se contó con las puntuaciones de severidad de sepsis como la evaluación de la falla orgánica secuencial (SOFA; del inglés, Sequential Organ Failure Assesment) [32] y la evaluación de la fisiología aguda y la salud crónica II (APACHE II; del inglés, Acute Physiology And Chronic Health Evaluation II) [33], los cuales son indispensables al momento de valorar un paciente con sepsis, pues no estaban disponibles de manera homogénea y estandarizada en los pacientes incluidos en la cohorte. Otra limitación fue la no disponibilidad de múltiples mediciones de proteína $C$ reactiva y procalcitonina para los individuos incluidos para realizar un análisis más detallado. Por último, no se ejecutó una valoración adecuada y uniforme de las comorbilidades como la enfermedad renal crónica, la cual se ha evidenciado en estudios previos como un factor asociado a niveles elevados de presepsina [15].

\section{Conclusiones}

En el presente trabajo la presepsina mostró un buen desempeño como ayuda diagnóstica temprana de la sepsis y fue consistente con los hallazgos de estudios previos. Además, este marcador aporta un valor agregado al diagnóstico de sepsis en comparación con los métodos tradicionales y de rutina en el laboratorio clínico. Asimismo, esta investigación documenta la experiencia de este novedoso biomarcador en un hospital de alto nivel de complejidad en miras a que en otras instituciones del mismo nivel se implemente, en particular, para los pacientes del servicio de urgencias y de unidades de cuidado intensivo.

Finalmente, es preciso realizar otros estudios con cohortes más amplias, que puedan evaluar el valor pronóstico y el seguimiento de la sepsis en instituciones locales para generar conocimiento que se pueda integrar a las guías de práctica clínica en el manejo integral de la sepsis. 


\section{Agradecimientos}

Al laboratorio clínico del Hospital Universitario de San Vicente Fundación en la realización de las pruebas. A Lina María Echeverri Toro, MD, Msc, por la asesoría clínica y científica del estudio. A Carlos Adrián Peñata Bedoya, MB, MSP, por la revisión y redacción del presente manuscrito.

Este estudio fue realizado con recursos del Hospital Universitario de San Vicente Fundación y las pruebas de presepsina financiadas por la empresa Vélez Lab. S.A.S.; no obstante, esta compañía no tuvo ninguna participación en el análisis de los resultados.

\section{Bibliografía}

1. Levy MM, Fink MP, Marshall JC, Abraham E, Angus D, Cook D, et al. 2001 SCCM/ESICM/ACCP/ATS/SIS International Sepsis Definitions Conference. Intensive Care Med 2003; 29: 530-538.

2. Bone RC, Balk RA, Cerra FB, Dellinger RP, Fein AM, Knaus WA, et al. Definitions for sepsis and organ failure and guidelines for the use of innovative therapies in sepsis. The ACCP/SCCM Consensus Conference Committee. American College of Chest Physicians/Society of Critical Care Medicine. Chest 1992; 101: 1644-1655.

3. Angus DC, Linde-Zwirble WT, Lidicker J, Clermont G, Carcillo J, Pinsky MR. Epidemiology of severe sepsis in the United States: analysis of incidence, outcome, and associated costs of care. Crit Care Med 2001; 29: 1303-1310.

4. Tanriover MD, Guven GS, Sen D, Unal S, Uzun O. Epidemiology and outcome of sepsis in a tertiary-care hospital in a developing country. Epidemiol Infect 2006; 134: 315-322.

5. Dellinger RP, Levy MM, Rhodes A, Annane D, Gerlach H, Opal SM, et al. Surviving Sepsis Campaign: international guidelines for management of severe sepsis and septic shock, 2012. Intensive Care Med 2013; 39: 165-228.

6. Bochud PY, Bonten M, Marchetti O, Calandra T. Antimicrobial therapy for patients with severe sepsis and septic shock: an evidence-based review. Crit Care Med 2004; 32: S495-512.

7. Rodriguez F, Barrera L, De La Rosa G, Dennis R, Duenas C, Granados M, et al. The epidemiology of sepsis in Colombia: a prospective multicenter cohort study in ten university hospitals. Crit Care Med 2011; 39: 1675-1682.
8. Restrepo MI, Dueñas C, González M, Ortiz G, Granados $\mathbf{M}$, Álvarez $\mathrm{C}$, et al. Consenso colombiano en sepsis. Infectio 2007; 11: 46-56.

9. Fischer JE, Bachmann LM, Jaeschke R. A readers' guide to the interpretation of diagnostic test properties: clinical example of sepsis. Intensive Care Med 2003; 29: 1043-1051.

10. Hernández-Bou S, Trenchs Sainz de la Maza V, Esquivel Ojeda JN, Gené Giralt A, Luaces Cubells C. Factores predictores de contaminación ante un hemocultivo con crecimiento bacteriano en Urgencias. An Pediatr 2015; 82: 426432.

11.Zhang J, Hu ZD, Song J, Shao J. Diagnostic Value of Presepsin for Sepsis: A Systematic Review and Meta-Analysis. Medicine (Baltimore) 2015; 94: e2158.

12. Faix JD. Biomarkers of sepsis. Crit Rev Clin Lab Sci 2013; 50: 23-36.

13. Cho SY, Choi JH. Biomarkers of sepsis. Infect Chemother 2014; 46: 1-12.

14. Yaegashi Y, Shirakawa K, Sato N, Suzuki Y, Kojika M, Imai S, et al. Evaluation of a newly identified soluble CD14 subtype as a marker for sepsis. J Infect Chemother 2005; 11: 234-238.

15. Shozushima T, Takahashi G, Matsumoto N, Kojika M, Okamura Y, Endo S. Usefulness of presepsin (sCD14ST) measurements as a marker for the diagnosis and severity of sepsis that satisfied diagnostic criteria of systemic inflammatory response syndrome. J Infect Chemother 2011; 17: 764-769.

16. Shirakawa K, Naitou K, Hirose J, Takahashi T, Furusako $\mathrm{S}$. Presepsin (sCD14-ST): development and evaluation of one-step ELISA with a new standard that is similar to the form of presepsin in septic patients. Clin Chem Lab Med 2011; 49: 937-939.

17. Carpio R, Zapata J, Spanuth E, Hess G. Utility of presepsin (sCD14-ST) as a diagnostic and prognostic marker of sepsis in the emergency department. Clin Chim Acta 2015; 450: 169-175.

18. Muller B, Becker KL, Schachinger H, Rickenbacher PR, Huber PR, Zimmerli W, et al. Calcitonin precursors are reliable markers of sepsis in a medical intensive care unit. Crit Care Med 2000; 28: 977-983.

19. Harbarth S, Holeckova K, Froidevaux C, Pittet D, Ricou B, Grau GE, et al. Diagnostic value of procalcitonin, interleukin-6, and interleukin-8 in critically ill patients admitted with suspected sepsis. Am J Respir Crit Care Med 2001; 164: 396-402.

20. Abbott Laboratories. C-reactive protein. Illinois, Estados Unidos. 2008. Disponible: http://www.ilexmedical. com/files/PDF/CRP_ARC_CHEM.pdf. Consultado: feb 2017.

21. Burtis CA, Ashwood ER. Tietz Textbook of Clinical Chemistry (ed 3a). Filadelfia, Estados Unidos: WB Saun- 
ders; 1998.

22. Mitsubishi Chemical Europe GmbH, LSI Medience Corporation. PATHFAST ${ }^{\mathrm{TM}}$ Presepsin. 2014. Disponible: http://www. pathfast.de/images/stories/download/PF_ Folder_SEPSIS_2014.pdf. Consultado: feb 2017.

23. Martin GS, Mannino DM, Eaton S, Moss M. The epidemiology of sepsis in the United States from 1979 through 2000. N Engl J Med 2003; 348: 1546-1554.

24. López-Vallejo CA. Epidemiología de la sepsis en la Fundación Cardioinfantil - Instituto de Cardiología - Bogotá. Tesis para optar al título de Especialista en Medicina Interna. Bogotá D.C., Colombia: Universidad del Rosario; 2009.

25. Julián-Jiménez A, Palomo-De Los Reyes MJ, OrtizDíaz-Miguel R, Pedrosa-Guerrero A, Parejo-Miguez R, Salcedo-Martínez R. Utilidad de la procalcitonina y la proteína $\mathrm{C}$ reactiva en el paciente con sepsis en urgencias. Emergencias 2009; 21: 23-27.

26. Enguix A, Rey C, Concha A, Medina A, Coto D, Dieguez MA. Comparison of procalcitonin with C-reactive protein and serum amyloid for the early diagnosis of bacterial sepsis in critically ill neonates and children. Intensive Care Med 2001; 27: 211-215.

27. Endo S, Suzuki Y, Takahashi G, Shozushima T, Ishikura $\mathbf{H}$, Murai A, et al. Usefulness of presepsin in the di- agnosis of sepsis in a multicenter prospective study. J Infect Chemother 2012; 18: 891-897.

28. Pertuz M Y, González R G, Acosta M S. Uso de biomarcadores de inflamación o infección en el diagnóstico de sepsis, en unidades de cuidados intensivos de Santa Marta, Colombia. Biosalud 2016; 15: 28-36.

29. Sánchez-González RA, Becerra-Victorio G, GrajalesAvendaño L, Canseco-Ávila LM. Frecuencia de microorganismos aislados de hemocultivos en un hospital de tercer nivel en el estado de Chiapas. Enf Inf Microbiol 2010; 30: 53-58.

30. Tong X, Cao Y, Yu M, Han C. Presepsin as a diagnostic marker for sepsis: evidence from a bivariate meta-analysis. Ther Clin Risk Manag 2015; 11: 1027-1033.

31. Povoa P, Coelho L, Almeida E, Fernandes A, Mealha $\mathbf{R}$, Moreira $\mathrm{P}$, et al. C-reactive protein as a marker of infection in critically ill patients. Clin Microbiol Infect 2005; 11: 101-108.

32. Minne L, Abu-Hanna A, de Jonge E. Evaluation of SOFA-based models for predicting mortality in the ICU: A systematic review. Crit Care 2008; 12: R161.

33. Knaus WA, Draper EA, Wagner DP, Zimmerman JE. APACHE II: a severity of disease classification system. Crit Care Med 1985; 13: 818-829.

Introduction: Presepsin is the soluble form of CD14 molecule, a glycoprotein expressed on the membrane surface of monocytes and macrophages and important to inflammatory response. Several studies suggest that presepsin measuring could be used as a good tool to early sepsis diagnosis. Objective: To determine the diagnostic and prognostic performance of presepsin in patients with clinical sepsis at a high complexity hospital from Medellin, Colombia. Materials and methods: A prospective, descriptive cohort study was performed in 60 patients diagnosed with clinical sepsis between March and December of 2012. Presepsin measurement was made at diagnosis time and after 24 and 72 hours. Blood culture, C-reactive protein and procalcitonin were also measured but only at the diagnosis time. Results: 98.3\% of patients had presepsin values suggestive of sepsis, with a significantly higher value in patients without clinical improvement (greater than $700 \mathrm{pg} / \mathrm{mL}$ ). Presepsin values greater than 1,000 pg / $\mathrm{mL} \mathrm{at}$ 0 and 72 hours of diagnosis was obtained in patients who died, but no significant differences compared to those who did not die were observed. C-reactive protein and procalcitonin showed increased values in most patients. Conclusions: The findings of this study are related to others where it is concluded that presepsin is a good marker of sepsis with an important prognostic value and greater specificity than other traditional biomarkers.

Key words: Sepsis, biomarkers, diagnosis, CD14 antigens. 